\title{
PRIMARY CARCINOMA OF THE AMPULLA OF VATER.
}

\author{
By F. Craven Moore, M.D., M.Sc., M.R.C.P., Assistant to the Pro- \\ fessor of Medicine and Lecturer in the Victoria University of \\ Manchester.
}

\section{(Plate VIII.)}

Carcinomata, originating in the mucous membrane lining the ampulla of Vater, the small common channel situate within the duodenal wall by which the common bile duct and the duct of Wirsung communicate with the duodenum, have conveniently, if somewhat empirically, been regarded as a class anatomically distinct from similar neoplasms originating in the mucous lining of the adjacent lower end of the common bile duct.

The ampulla of Vater, morphologically regarded, would appear to be the true termination of the common bile duct; the mucous membrane of the two channels is anatomically continuous and structurally identical, and neoplasms originating in them have a common morphology.

The discrimination between primary neoplasms in these contiguous situations is based entirely on their relative frequency and on their diverse obstruent effects, the ampullary carcinoma implicating both the common bile duct and the duct of Wirsung. The relative incidence of primary carcinoma of the common bile duct, the juxtaampullary segment being its most frequent seat, is very small, but compared with that of primary carcinoma of the ampulla it is already considerable. My own experience includes three examples of the former and one of the latter condition; and on the more convincing basis of recorded cases it is noteworthy that, whilst in 1901 Devic and Gallavardin were able to collate fifty-five examples of primary carcinoma of the extra-hepatic bile ducts, it is the opinion of Hanot and also of Rolleston that a small proportion only of the recorded cases of ampullary carcinoma really merit this appellation, the absolute number of veritable examples being probably not greater than eight or nine.

The case here presented was in the Manchester Royal Infirmary, 
under the care of Dr. A. T. Wilkinson, to whom I am indebted for permission to make this use of it.

CASE.-A charwoman, æt. 43, complained of weakness, wasting, jaundice, and vomiting. At the age of 21 years she had an attack of jaundice, without. pain; this shortly disappeared, leaving her in good health, which she continued to enjoy until eight months ago; then she began to suffer from loss of appetite, pain after food, with occasional vomiting, and loss of energy. Five months later, these disturbances having continued with increasing severity, she gradually becanle jaundiced; vomiting now occurred several times a day; diarrhoa was constantly present, and the loss of strength accompanied by wasting was so marked that she became unable to follow her occupation.

On admission to hospital, the patient was emaciated and deeply jaundiced, the stools were acholic, the urine contained much bile and a small amount of albumin. The liver was enlarged, extending 2 inches below the costal margin; it was firm, and free from any irregularity. The gall bladder, much enlarged and tense, was easily palpable.

With increasing cachexia, she became comatose, and died nine months from the first manifestation of ill-health.

Autopsy.-The body was much emaciated, the skin and visible mucous membranes were deeply jauncliced. The subcutaneous tissue was almost devoid of fat.

Circulatory system.-The pericardium was normal ; the heart, weight 61 oz., presented in a typical fashion the characters of brown atrophy; the arteries and veins were normal.

Respiratory system.-Pleuræ normal; both pleural cavities contained some excess of bile-stained serous fluid; the lungs were the seat of œedema, and postero-inferiorly were congested and friable; the lower lobe of the right lung was, in addition, partially collapsed by elevation of the right dome of the diaphragm. The air passages were normal.

Digestive system.- Esophagus normal ; the stomach was greatly dilated, the greater curvature reaching below to the pubic symphisis; it contained a large quantity of a grey fluid material; its mucosa was covered with a thick layer of tenacious mucus. The dilatation involved also the pylorus, which was otherwise normal, and the first part of the duodenum. The rest of the duodenum and the small intestine were normal; the large intestine was. somewhat contracted.

The peritoneum, visceral and parietal, was normal.

The liver, weight 49 oz., was somewhat enlarged, of normal shape, dark green in colour, smooth and firm; on section, the hepatic parenchyma had a variegated appearance from the coincidence of biliary and fatty infiltration; the intra-hepatic bile ducts were much dilated and occupied by a thick tenacious bile-stained mucus; the blood vessels were normal.

The gall bladder was much enlarged, being distended with bile-stained mucus; the cystic duct was normal; the hepatic ducts and the common bile duct were greatly dilated, the latter on being opened readily admitted the little finger, which could be passed along it by the head of the pancreas to the wall of the duodenum, where it contracted abruptly like the end of a test tube. In no part of the biliary channels were any calculi found.

The pancreas, of normal size and shape, was firmer in consistency; on section, the duct of Wirsung was seen to be dilated.

The spleen, weight $4 \mathrm{oz}$., was of normal appearance.

The uro-genital system. - The kidneys, each $4 \mathrm{oz}$. in weight, were the seat of biliary infiltration and early parenchymatous inflammation; the urinary passages and the genital organs were normal.

The pancreas along with the duodenum and the extra-hepatic bile ducts being removed from the body, the following condition was found:-On laying 
open the duodenum the bile papilla appeared much enlarged, of a truncated conical shape, and was firm to the touch; the mucosa covering it had a perfectly normal appearance; its orifice was patent and slightly enlarged, but it was found impossible to pass a probe further than into the orifice.

A coronal section through the dundenum and the head of the pancreas in the plane of the bile papilla (Plate VIII. Fig. 1) showed that the enlargement of the latter was due to the presence of a mass of firm, greyish-white tissue, occupying the position of the ampulla of Vater. Probes passed into the common bile duct and into the duct of Wirsung were not arrested until the point of their confluence was reached, and here the obstruction, as indicated by the dilatation of the two ducts, was complete.

About the head of the pancreas there appeared to be considerable hyperplasia of the peri-glandular connective tissue.

The adjacent lymphatic glands were slightly enlarged and infiltrated with bile, but neither here nor elsewhere in the bolly was there any macroscopic evidence of metastasis.

Microscopic Appearaxces. - Sections through the bile papilla and adjacent portion of the pancreas, in the same plane as the macroscopic section, failed to reveal any continuous line of mucous membrane such as would have been present, delimiting internally the wall of the ampulla, had that channel been merely invaded by a mass of new growth proceeding from adjacent structures; they showed the situation of the ampulla, increased in extent, to be occupied by a mass of neoplastic tissue penetrating between the muscular bundles of the wall of the duodenum on the one side, and on the other delimited from the pancreas by a considerable layer of fibrous tissue.

In structure the neoplastic tissue had the characters of a columnar-celled carcinoma, consisting of a richly cellular connective tissue stroma and elongated and irregular duct-like spaces, lined by a single layer of columnar cells (Plate VIII. Figs. 2 and 3).

The liver was the seat of marked biliary infiltration, the bile ducts, even to their intracellular ramifications, being greatly dilated; about the small interlobular ducts the connective tissue was increased in amount, and more cellular than normal, while the larger branches presented the changes of early catarrhal inflammation. In no part of the liver was there found any evidence of metastasis. The gall bladder and the extra-hepatic ducts showed only catarrhal inflammation of their mucous lining, of slight degree.

The pancreas presented considerable hyperplasia of the interlobular stroma ; the gland tissue, including the islands of Langerhans, was normal, and the ducts were dilated and the seat of catarrhal inflammation.

The enlarged lymphatic glands were congested and infiltrated with bile, but presented no signs of neoplastic invasion.

The case is remarkable by reason of the contrast between the marked cachexia noted during life and the insignificance of the neoplastic process as determined post-mortem. Of the propriety of classing it as an example of carcinoma originating in the mucosa of the ampulla of Vater there can, I think, be little doubt; the anatomical relations rendered its differentiation from similar neoplasms of the anatomical termination of the common bile duct, and of the mucous membrane covering the duodenal surface of the biliary papilla, peculiarly easy, while its structure rendered confusion with carcinoma of the head of the pancreas impossible.

The number of anatomically distinct neoplasms which may obtain in this particular part of the digestive system is very striking; within 
an area having a radius no greater than one-quarter of an inch one may find columnar-celled carcinomata originating from the mucosa covering the duodenal surface of the biliary papilla, from that lining the ampulla, the lower end of the common bile duct, and, it is said, also from that of the termination of the duct of Wirsung, and a spheroidal-celled carcinoma from the gland tissue of the head of the pancreas.

All these neoplasms are, however, comparatively infrequent; two examples of the first or duodenal type have come under my own observation and offered no difficulties in post-mortem diagnosis, three of the common bile duct type, and several of the head of the pancreas. Carcinoma of the duct of Wirsung I have not seen or recognised.

The differentiation between carcinoma of the common bile duct and of the ampulla of Vater may call for minute examination, and the following features of one of the examples of the former which came under my notice will indicate the futility of instituting a discriminating inquiry into the value of the claims of many of the cases recorded as primary ampullary carcinomata for that distinction, and perhaps may prove equally disastrous to the utility of such anatomical differentiation.

The conditions found at the autopsy pointed to malignant obstruction of the common bile duct about its lower end. On opening the duodenum, the biliary papilla was seen to be much enlarged and conical in form; its orifice was patent; on section, the ampulla was found to be occupied by new growth, and the last quarter of an inch of the common bile duct was completely invested by an annular growth extending to and occluding its lumen; the duct of Wirsung was not dilated. Microscopically, it was found that between the mass of new growth occupying the cavity of the ampulla and the muscular coat of the duodenum a continuous layer of mucous membrane was present, constituting the lining of the ampulla, and which presented no manifestation of neoplastic change.

In this case the new growth, commencing in the lower end of the common bile duct, had fungated into and distended the contiguous ampulla.

In primary carcinoma of the ampulla of Vater the differential features appear to consist in the presence of neoplastic change in its mucous lining, the absence of such change from the lower end of the common bile duct, and in obstructive dilatation not only of the latter, but also of the duct of Wirsung.

Of the recorded cases, few only offer such details as permit of this differentiation, and beyond the cases described by Hanot, who is probably responsible for the recognition of the ampullary carcinoma as a distinct class, by Halstead, Rolleston, and Havilland Hall, it is quite uncertain to what category the majority belong. 


\section{REFERENCES.}

Jevic and Gallavardis - Rev de méd., Paris, 1901, tome xxi. p. 557.

Hanot, v. . . . . . Arch. gén. de méd., Paris, 1896, tome clxxviii. p. 547.

HaLl . . . . . . . Lancet, London, 1902, vol. i. p. 1102.

Halstead . . . . . Johns Hopkins Hosp. Bull., Baltimore, 1900, January.

RoLleston . . . . . Lancet, London, 1901, vol. i. p. 467.

\section{DESCRIPTION OF PLATE VIII.}

Fic. 1.-Coronal section through the second part of the duodenum and the head and body of the prancreas, in the plane of the biliary papilla. In the right of the figure is a part of the body of the pancreas, which has been ent away anteriorly to expose the dilated duct of Wirsung; the head of the pancreas, joined to the body by the narrower neck, is seen occupying the concavity of the duodenum, the cut edge of which is recognised by its muscular coat. From about the centre of the head of the pancreas, projecting into the lumen of the duodenum, where there is a break in the continuity of the muscular coat of the latter, is the enlarged biliary papilla ; the mncosa covering it is normal, and the enlargement is seen to be due to a mass of new growth in the situation of the ampulla. Above the head of the pancreas, to the right of the opened duodenum, is the dilated common bile duct, also laid open. Below the head of the pancreas is a mass of hyperplastic tibrous tissue.

Fig. 2.-Showing the structure of the new growth ocenpying the position of the ampulla of Vate:. $(\times 125$.

Fig. 3.-Same as Fig. 2. $(\times 500$. 


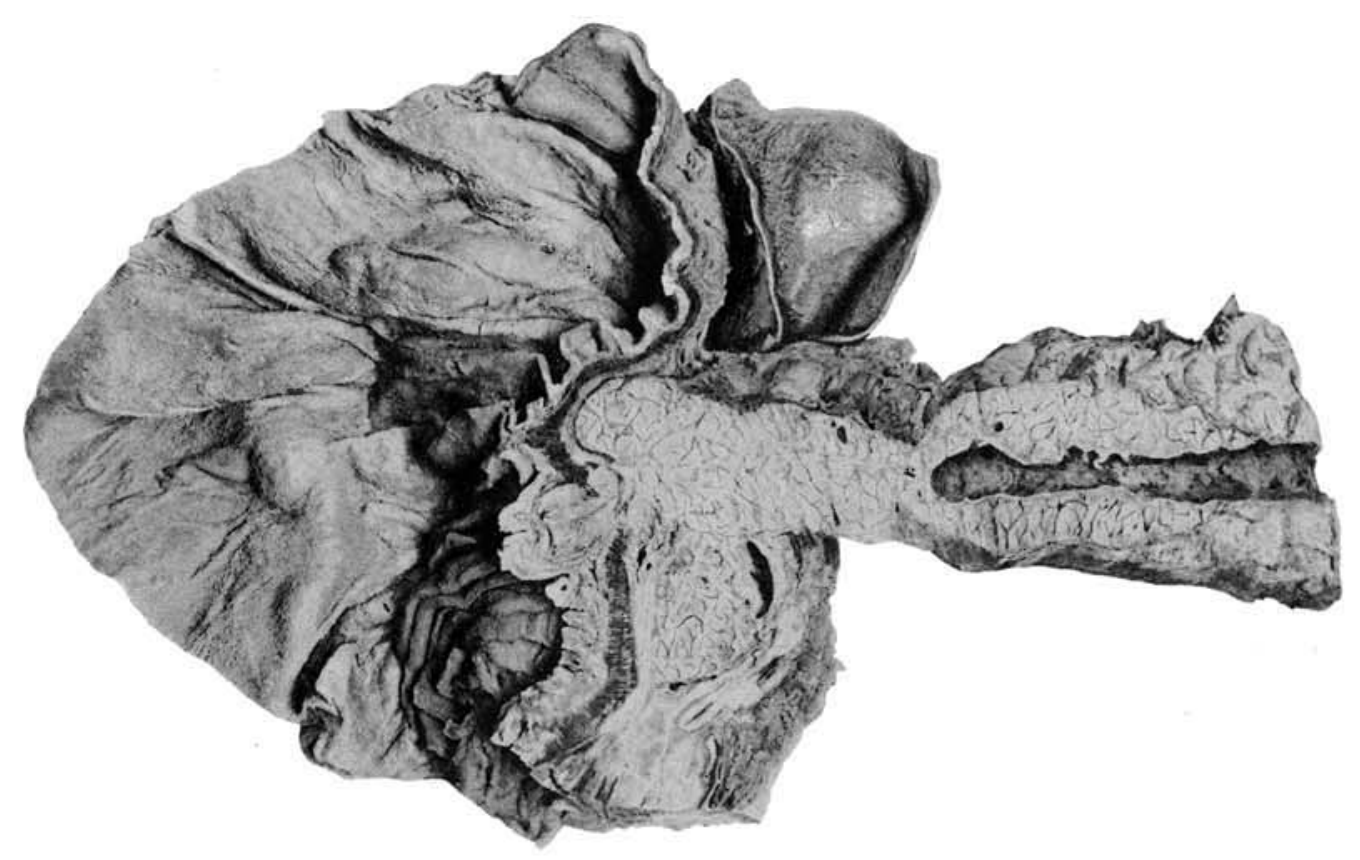

Fig. 1.

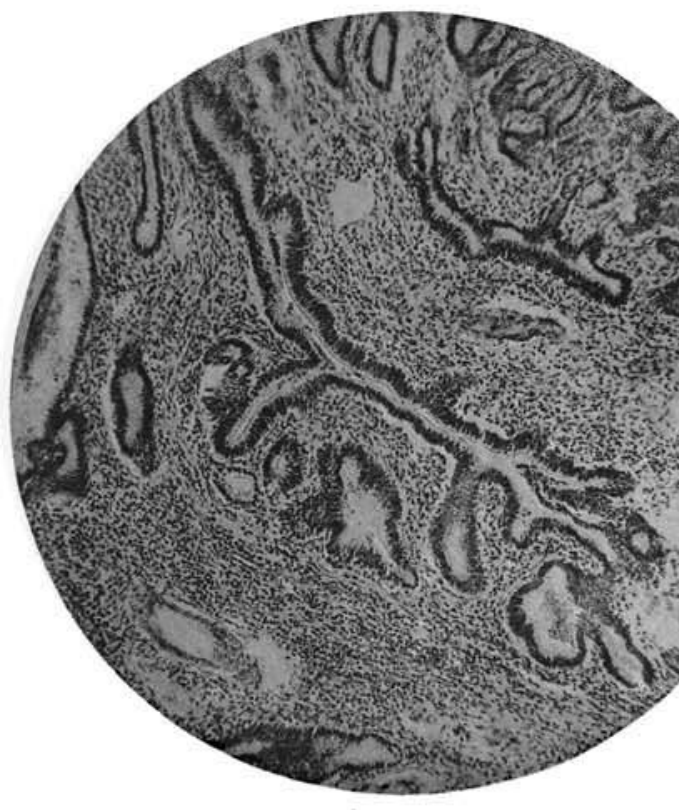

FIG. 2.

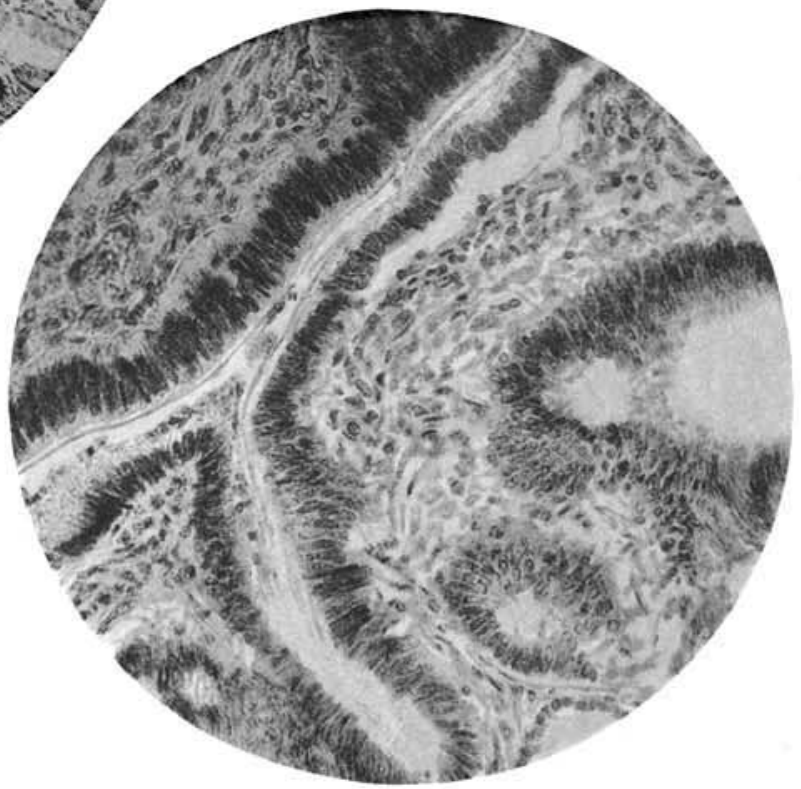

Fig. 3. 